\title{
Single-chip optical beam forming network in LPCVD waveguide technology based on optical ring resonators
}

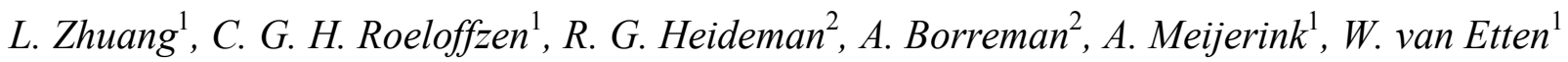 \\ ${ }^{1}$ University of Twente, Faculty of Electrical Engineering, Mathematics and Computer Science, \\ Telecommunication Engineering group, PO Box 217, 7500 AE, Enschede, The Netherlands
}

${ }^{2}$ LioniX B.V., PO Box 456, 7500 AH, Enschede, The Netherlands

\begin{abstract}
Optical ring resonators (ORRs) can be used as continuously tunable delay elements in a beam forming network for a phased-array antenna system. The bandwidth of such delay elements can be enhanced by cascading multiple ORRs. When delays and splitting/combining circuitry are integrated in one optical circuit, an optical beam forming network (OBFN) is obtained. In this paper, the principles of optical beam forming using ORRs are explained and demonstrated, by presenting measurements on a $1 \times 4$ OBFN chip, realized in LPCVD waveguide technology. To our knowledge, this is the first single-chip demonstration of continuous optical beam forming.
\end{abstract}

Index Terms - Delay filters, LPCVD waveguide technology, microwave photonics, optical beam forming, optical ring resonators, optical signal processing.

\section{INTRODUCTION}

Beam forming in wireless transmission can be achieved by means of a phased-array antenna, consisting of multiple antenna elements, a corresponding number of delay elements and splitting/combining circuitry. The geometry of the antenna array and the values of the delays determine the beam pattern of the smart antenna.

The delay elements need to be continuously tunable in order to achieve continuous beam angle control. Moreover, in order to support relatively broadband radio frequency (RF) signals, the delay elements should have a flat magnitude response and a linear phase response in the corresponding frequency range.

A continuously tunable delay element can be realized in optics, by cascading an electro-optic converter (a modulated laser), an optical delay element and an optoelectrical converter (a photodetector). The optical delay element can be realized as an optical integrated circuit containing a straight waveguide and multiple optical ring resonators (ORRs). The effective delay that an RF signal experiences when it passes through this cascade is determined by the group delay of the optical delay element, which can be controlled by tuning the coupling coefficients between the waveguide and the ORRs, and the round-trip phase shifts of the ORRs [1]-[5].

When the signal processing circuitry is also implemented in the optical domain, by integrating multiple delay elements and optical splitters/combiners on a single optical chip, an optical beam forming network $(\mathrm{OBFN})$ is obtained.

This paper demonstrates the advantages of integrated optical beam forming using ORRs, by explaining its theoretical principles, and by describing some measurement results on an actual $1 \times 4$ OBFN chip, realized in LPCVD planar optical waveguide technology.

Section II explains the principles of ORR-based OBFN subsystems. The design and realization of the actual device are described in Section III, followed by device characterization in Section IV. Conclusions are formulated in Section V.

\section{PRINCIPLES OF RING RESONATOR-BASED OPTICAL BEAM FORMING NETWORKS}

\section{A. Optical ring resonator (ORR) delays}

Delay elements in beam forming systems can be implemented by means of optical ring resonators (ORRs). Suppose we have a lossless ORR with roundtrip time $T$ and additional round-trip phase-shift $\phi$, which is coupled parallel to an optical waveguide, with power coupling coefficient $\kappa$. Then the waveguide will act as an optical all-pass filter, with group delay response [1]-[5]

$$
\tau_{\mathrm{g}}(f)=\frac{\kappa T}{2-\kappa-2 \sqrt{1-\kappa} \cos (2 \pi f T+\phi)} .
$$

Obviously, the group delay response is periodic, with period $1 / T$, also called free spectral range (FSR). Each period of the group delay response shows a bell shape, centered at a resonance frequency of the ring, which depends on $\phi$. The peak delay depends on the coupling coefficient $\kappa$, so that the value of the peak delay can be continuously tuned by varying $\kappa$. Hence continuous beam angle steering can be achieved. The optical bandwidth of the delay element (the width of the bell shape) is more or less inversely proportional to the peak delay. As a result, a single-ring delay element shows a trade-off between peak delay and bandwidth [1],[3]-[5]. 


\section{B. Multi-ring delay elements}

For a given delay tuning range, the corresponding minimum bandwidth will in general not suffice for providing time delay behavior in a broadband phasedarray antenna system. The bandwidth of the delay element can be increased, however, by cascading multiple ORR sections, as illustrated in the inset of Fig. 3 . Since the group delay responses of the individual sections simply add up, the total group delay response can be flattened by tuning the ORRs to different resonance frequencies $f_{1}, f_{2}$ and $f_{3}$. This is illustrated in Fig. 1.

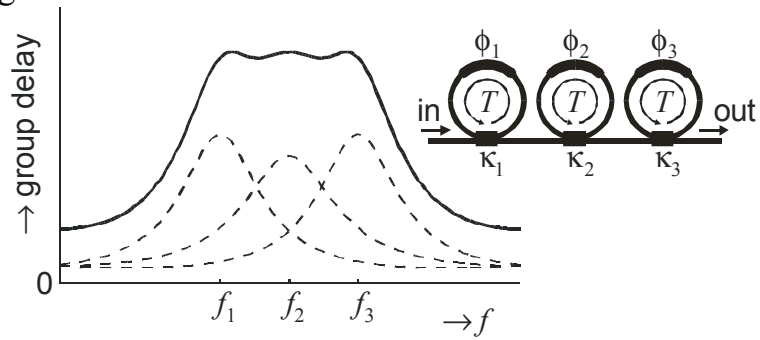

Fig. 1. Theoretical group delay response of three cascaded ORR sections. The dashed lines represent the group delay responses of the individual sections. Inset: cascade of three ORRs with round-trip times $T$, additional round-trip phaseshifts $\phi_{i}$ and power coupling coefficients $\kappa_{i}$.

The bandwidth of a multi-stage ORR-based delay element can be extended by adding more sections, and the peak-peak ripple of the group delay response in the flattened frequency band can be decreased by decreasing the difference between the resonance frequencies of the rings. As a result, multi-stage ORR-based delay elements show a trade-off between peak delay, optical bandwidth, relative group delay ripple and number of rings [1],[3][5]. Measurements on a 3-ring optical delay device realized in LPCVD waveguide technology [6] were presented in [5], showing good agreement with (1).

\section{Optical beam forming network (OBFN)}

The complete beam forming operation can be integrated into one optical chip by combining multiple ORR-based delay elements and additional optical splitters (in case of a transmitter array) or combiners (in case of a receiver array) in a so-called optical beam forming network (OBFN).

Integrating this functionality into one optical chip has many advantages, like compact size, light weight, and intrinsic electromagnetic compatibility. Moreover, integration on chip facilitates coherent optical combining, so that only one laser and one detector (and multiple external optical modulators) are required in a phased-array receiver system [7].

Several topologies are possible for structuring such OBFN. The most straightforward topology is the parallel OBFN, which consists of parallel delay elements and an optical splitter/combiner. In this paper, however, we will consider a binary tree OBFN topology, which is shown in Fig. 2. Although this topology requires an equal number of delay elements as the parallel topology, it can be verified that their tuning ranges can be smaller than in the case of a parallel topology. Consequently, the total required number of rings for a binary tree-based OBFN will be smaller than for a parallel OBFN.

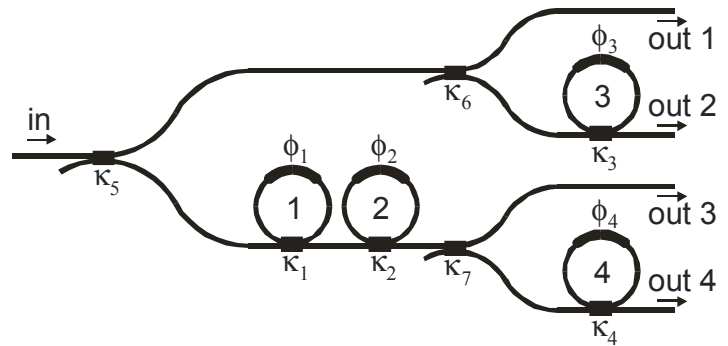

Fig. 2. Binary tree-based $1 \times 4$ optical beam forming network for a phased-array transmitter system.

\section{DEVICE DESIGN AND REALIZATION}

\section{A. Design}

The actual design of the $1 \times 4 \mathrm{OBFN}$ has been divided in the design of elementary basic building blocks: bends, Mach-Zehnder interferometers (MZIs) for the tunable coupling and splitting function, and the tapered waveguide end-face. Individual building blocks have been analyzed and simulated by commercially available software packages (Phoenix BV). Next, the most promising building blocks have been selected and fabricated using the TripleX ${ }^{\mathrm{TM}}$ technology [6]. This resulted into an optimal $1 \times 4$ OBFN which uses bends with a radius of $700 \mu \mathrm{m}$, and MZIs with directional coupler lengths of $150 \mu \mathrm{m}$ and an end-face waveguide taper that is tapered from a width of $1.5 \mu \mathrm{m}$ and a height of $30 \mathrm{~nm}$ to a width of $2 \mu \mathrm{m}$ and a height of $220 \mathrm{~nm}$, over a length of $300 \mu \mathrm{m}$. The actuation of the couplers, splitters and phase shifters is done by heaters, allowing tuning of the resonance frequency of the ORR, tuning of the splitting ratio, and tuning of the delay within $1 \mathrm{~ms}$.

The composition of the actual $1 \times 4$ OBFN is extended to a $1 \times 8$ OBFN by adding an extra stage. Each $1 \times 4$ OBFN can be measured independently by using the extra ports of the MZI-based tunable couplers.

\section{B. Realization}

A number of $1 \times 8$ OBFN devices have been realized using the Triplex ${ }^{\mathrm{TM}}$ technology. Fig. 3 shows a photograph of a realized $1 \times 8$ OBFN chip. The chip length is $4.85 \mathrm{~cm}$ and the chip width is $0.95 \mathrm{~cm}$.

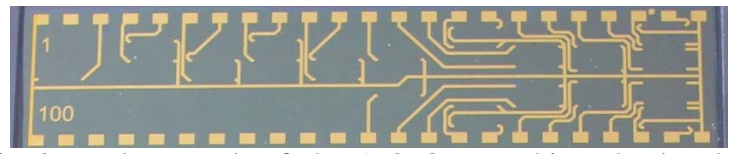

Fig. 3. Photograph of the $1 \times 8$ OBFN chip. The bondpads and electrodes are clearly visible. 
A single $1 \times 4$ OBFN as depicted in Fig. 2 requires 11 heaters and therefore at least 11 electrodes and one ground electrode to drive the heater elements.

\section{MEASUREMENTS}

\section{A. Measurement approach and setup}

The optical group delay responses at the outputs 2, 3 and 4 of a $1 \times 4$ OBFN chip have been measured by means of the phase-shift method, using the setup shown in Fig. 4.

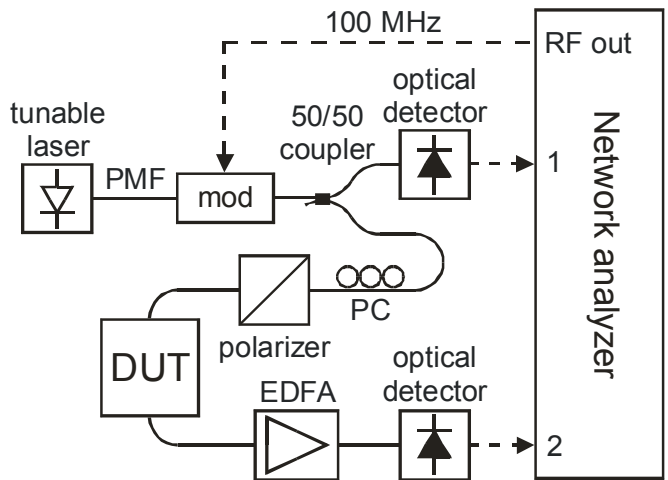

Fig. 4. Group delay measurement setup. DUT: Device under test, EDFA: erbium-doped fiber amplifier, mod: external MachZehnder intensity modulator, PC: polarization controller, PMF: polarization-maintaining fiber.

The key apparatus in this measurement setup is the Agilent PNA network analyzer. It generates an electrical $\mathrm{RF}$ signal with a fixed frequency of $100 \mathrm{MHz}$, which modulates the monochromatic light from the Santec tunable laser by means of an external $\mathrm{LiNiO}_{3}$ MachZehnder-based intensity modulator. The modulated optical signal is splitted by means of a fiber splitter. One part is detected and directly led back to the network analyzer. The other part is re-polarized and coupled into the device under test (DUT), such that the $1 \times 4$ OBFN is investigated for TE-polarized light. The three output powers are subsequently measured by manually changing the output ports. An erbium-doped fiber amplifier is used to boost the optical power, which is measured by means of a second detector and led to the network analyzer.

The network analyzer measures the RF phases $\varphi_{1}(\lambda)$ and $\varphi_{2}(\lambda)$ of the output signals of the optical detectors for different laser wavelengths $\lambda$. The group delay response is estimated from these results by calculating

$$
\tau_{\mathrm{g}}(\lambda)=\frac{\varphi_{1}(\lambda)-\varphi_{2}(\lambda)}{2 \pi f_{\mathrm{RF}}},
$$

where $f_{\mathrm{RF}}$ is the frequency of the electrical signal (100 $\mathrm{MHz}$ in our case). Note that this is a relative group delay response: it is biased by an eventual path length difference between the two paths from the 50/50 coupler to the inputs of the network analyzer.
The average powers at the outputs of the OBFN chip were measured using an HP 81532A Power Sensor (not shown in Fig. 4).

\section{B. Results}

Using this setup the group delays at the outputs 2, 3 and 4 of the OBFN chip (see Fig. 2) were measured over one FSR of the response, which is about $14 \mathrm{GHz}$ (proven by measurement), corresponding to a ring circumference of $1.2 \mathrm{~cm}$ and group index of 1.8. The measured waveguide loss is $« 1 \mathrm{~dB} / \mathrm{cm}$. Measurements were carried out with different settings for the heating elements. The results are shown in Fig. 5.

Fig. 5(a) and (b) are the group delay responses at output 2 and 3, which have one ring and two cascaded rings in their delay paths, respectively. The inherent trade-off between the delay bandwidth and peak delay value is demonstrated, which is in accordance with the theory in Section II. Fig. 5(c) demonstrates that the group delay response at output 4 (three cascaded rings in the delay path) is the sum of the two individual responses of two delay sections: two cascaded rings (ring 1 and 2) and ring 4 . Note that, due to the measurement approach, the individual group delay responses of two delay sections consist of the contributions of ring resonators and a certain length of straight waveguide lying between the input and output of each delay path. Therefore the peak delay of three rings is slightly lower than the sum of the two individual peak delays.

As a $1 \times 4$ OBFN, the four outputs are required to have four particular individual delay values over a certain frequency band, in order to satisfy the condition of beam forming. Fig. 5(d) demonstrates the simultaneous group delay responses of outputs 2,3 , and 4 of the $1 \times 4$ OBFN chip with output 1 as the zero delay reference. The coupling coefficients and round-trip phase-shifts of the rings are tuned such that three linearly increasing delays are obtained over $1.5 \mathrm{GHz}$ bandwidth, with a largest delay value of approximately $0.5 \mathrm{~ns}$ (corresponding to 15 $\mathrm{cm}$ of physical distance in air) and delay ripple of approximately $20 \mathrm{ps}(6 \mathrm{~mm})$.

\section{CONCLUSION}

A novel ring resonator-based optical beam forming network for broadband phased-array antenna applications has been proposed and realized in low-cost CMOS compatible LPCVD planar optical waveguide technology.

Group delay responses at different outputs of a $1 \times 4$ OBFN chip have been measured, in terms of the response of one independent ring, two cascaded rings, and the combination of these. The measurement results are in good agreements with theory.

Four-element optical beam forming is demonstrated with a maximum delay of $0.5 \mathrm{~ns}$ and a bandwidth of 1.5 $\mathrm{GHz}$. To our knowledge, this is the first single-chip demonstration of continuous optical beam forming. 


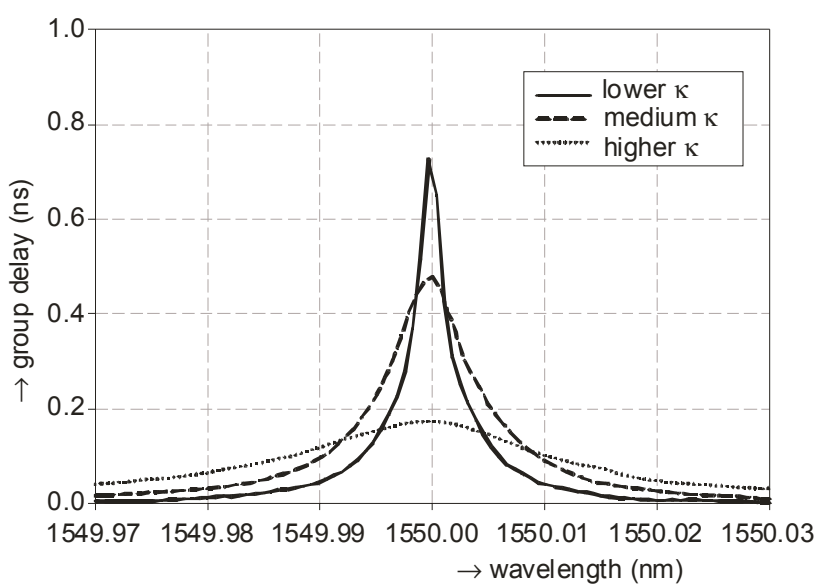

(a)

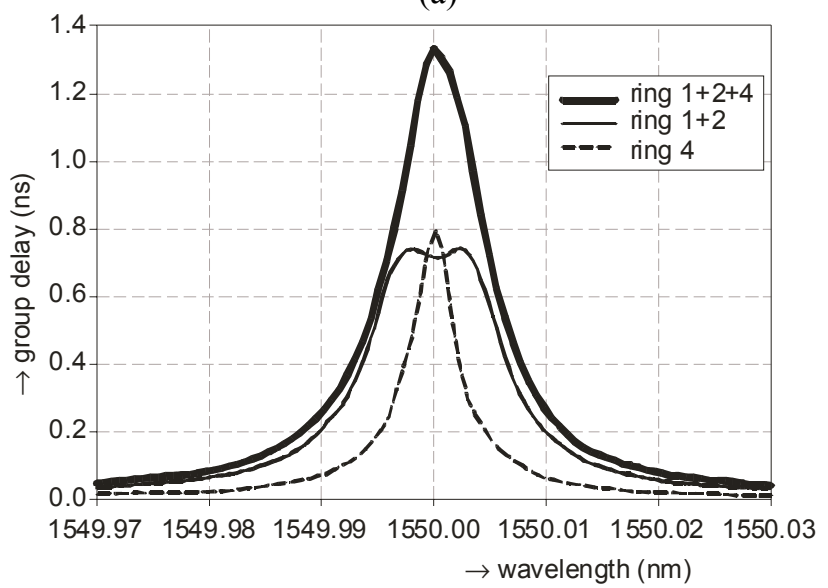

(c)

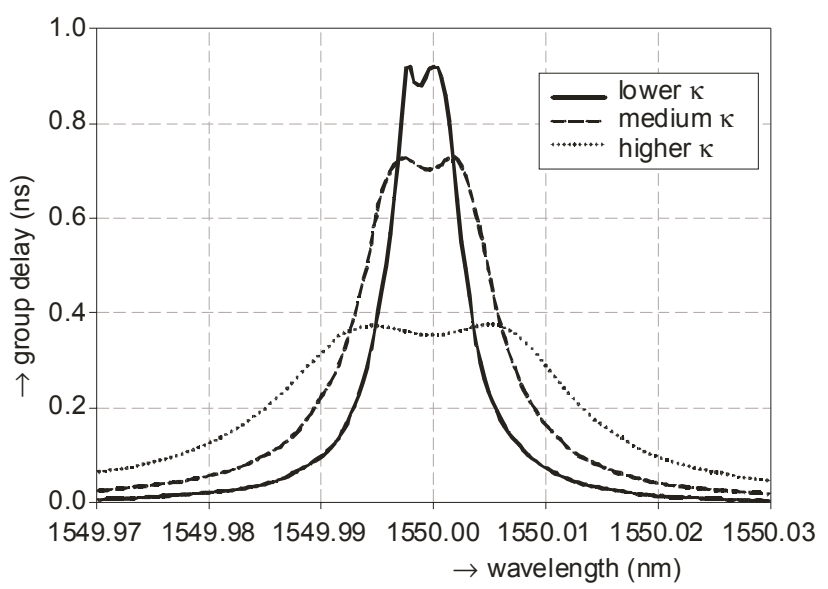

(b)

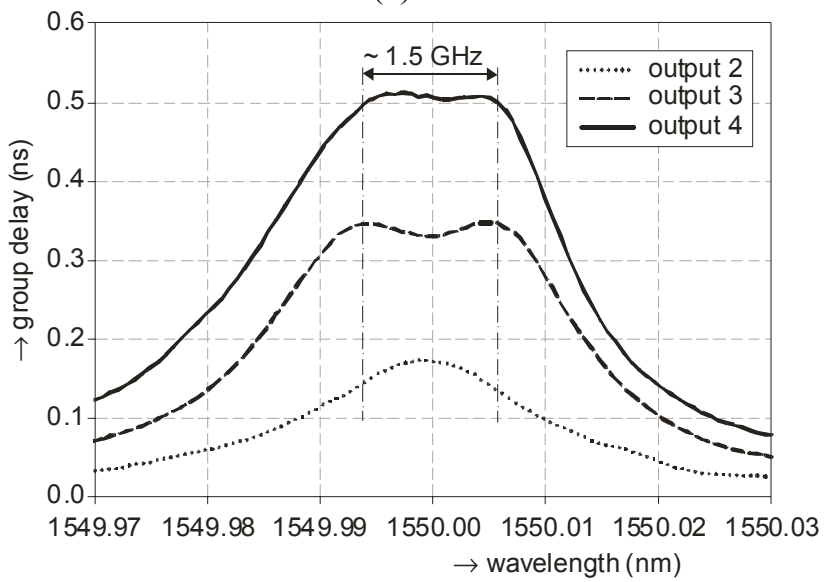

(d)

Fig. 5. Measurement results of different outputs of the $1 \times 4$ OBFN chip (as shown in Fig. 2). (a) and (b) show group delay responses of output 2 and 3, respectively, with various peak delay values; (c) shows the group delay responses of output 4 and the two individual delay sections in the corresponding delay path; (d) shows the simultaneous group delay response of output 2,3 , and 4 .

\section{ACKNOWLEDGEMENTS}

This work was part of the Broadband Photonic Beamformer project and the IO-BFN project, both supported by the Dutch Ministry of Economic Affairs, SenterNovem project number IS052081 and NIVR project number PEP 61424, respectively.

Robert Wijn and Rineke Groothengel are acknowledged for technical assistance during the device fabrication, and Eduard Bos is acknowledged for technical assistance during the laboratory work.

\section{REFERENCES}

[1] G. Lenz, B. J. Eggleton, C. K. Madsen, R. E. Slusher, "Optical delay lines based on optical filters," IEEE J. Quantum Electron., Vol. 37, No. 4, April 2001, pp. 525-532.

[2] J. E. Heebner, V. Wong, A. Schweinsberg, R. W. Boyd, D. J. Jackson, "Optical transmission characteristics of fiber ring resonators," IEEE $J$. Quantum Electron., Vol. 40, No. 6, June 2004, pp. 726-730.
[3] M. S. Rasras et al., "Integrated resonance-enhanced variable optical delay lines," IEEE Photon. Technol. Lett., Vol. 17, No. 4, April 2005, pp. 834-836.

[4] L. Zhuang, C. G. H. Roeloffzen, W. van Etten, "Continuously tunable optical delay line," Proc. of the 12th IEEE/CVT Symp. in the Benelux, Enschede, The Netherlands, November 2005.

[5] C. G. H. Roeloffzen, L. Zhuang, R. G. Heideman, A. Borreman, W. van Etten, "Ring resonator-based tunable optical delay line in LPCVD waveguide technology," Proc. of the 9th IEEE/LEOS Symp. in the Benelux, Mons, Belgium, December 2005, pp. 79-82.

[6] R. G. Heideman, A. Melloni, M. Hoekman, A. Borreman, A. Leinse, F. Morichetti, "Low loss, high contrast optical waveguides based on CMOS compatible LPCVD processing: technology and experimental results," Proc. of the 9th IEEE/LEOS Symp. in the Benelux, Mons, Belgium, December 2005, pp. 71-74.

[7] A. Meijerink, C. G. H. Roeloffzen, L. Zhuang, D. A. I. Marpaung, W. van Etten, "Signal processing in a phased-array antenna receiver system using a ring resonator-based optical beam forming network," Submitted to MWP 2006, Grenoble, France, 3-6 October 2006, this issue. 